\title{
PAPEL DE LAS EMPRESAS Y DE LOS ESTADOS EN LA DEBIDA DILIGENCIA EN DERECHOS HUMANOS
}

\section{THE ROLE OF THE STATES AND COMPANIES IN HUMAN RIGHTS DUE DILIGENCE}

\author{
Pilar IbáÑEZ-M.* \\ Viviana OrdoñEZ-S.**
}

\begin{abstract}
Para citar este artículo / To cite this article
Ibáñez-M., P. \& Ordóñez-S., V., Papel de las empresas y de los Estados en la debida diligencia en derechos humanos, 24 International Law, Revista Colombiana de Derecho Internacional, 219-246 (2014).
\end{abstract}

doi:10.11144/Javeriana.IL14-24.pedd

* Abogada litigante de la Pontificia Universidad Javeriana de la Universidad del País Vasco. Directora del Centro Internacional de Pensamiento e Investigación en Derecho (CIPID). Miembro del equipo asesor y jurídico de ILOCAD y la Fundación Internacional Baltasar Garzón. Trabajó en la MAPP/OEA, Dechert LLP, CNRR, BBVA, MSC. Tiene una maestría en Relaciones Exteriores y Derecho Internacional Público. Correo electrónico: pibanezabogados@gmail.com

** Abogada de la Pontificia Universidad Javeriana. Abogada del Centro Internacional de Pensamiento e Investigación en Derecho (CIPID). Asesora legal de la Fundación para la Libertad de Prensa (FLIP). Correo electrónico: ordonezsalazar.viviana@gmail.com 


\section{RESUMEN}

La relación de las empresas con los derechos humanos ha ido tomando cada vez más fuerza y en ese sentido se hace necesario precisar conceptos y apreciaciones sobre cuáles responsabilidades le son atribuibles a las empresas y cuáles a los Estados. Así, en el presente artículo las autoras buscan acercarse al concepto de debida diligencia, consagrada en los principios rectores sobre las empresas y los derechos humanos de John Ruggie, la cual recae en cabeza de las empresas en cuanto al respeto y protección de los derechos humanos, al tiempo que se quiere mostrar cuáles aspectos de este deber son responsabilidad de los particulares y cuáles del Estado. Por tanto, se expondrán, a lo largo del artículo, (I) el concepto de debida diligencia; (II) cómo elaborar un proceso de debida diligencia en el marco de derechos humanos; (III) el desarrollo e implementación de procesos de debida diligencia en derechos humanos; (IV) límites a la debida diligencia de las empresas; y (V) precisar en materia de debida diligencia, cuáles son las obligaciones del Estado y cuáles sus límites. A modo de conclusión, las autoras buscan dar recomendaciones que permitan tanto a empresas comoa Estados, un cumplimiento efectivo de sus obligaciones y responsabilidades.

Palabras clave autoras: Debida diligencia, empresas, derechos humanos, responsabilidad estatal, principios rectores de John Ruggie, Pacto Global, rol de la empresa.

Palabras clave descriptores: Contrato de trabajo, derechos humanos, responsabilidad del estado, Ruggie, John Gerard, 1944. 


\begin{abstract}
The relation between companies and Human Rights has been gaining more and more strength and in that sense it is necessary to specify concepts and insights about what responsibilities we are attributable to companies and what States. So, in this article the authors seek to approach the concept of due diligence, enshrined in John Ruggie's Guiding Principles, which lies at the head of companies in terms of respect and protection of human rights, while also wants to show which aspects of that duty are the responsibility of individuals and which are the responsibility of the State. Therefore, it will be discussed throughout the article (I) the concept of due diligence, (ii) how to develop a process of due diligence in the context of human rights, (iii) the development and implementation of due diligence processes and human rights, (iv) limits on the due diligence of companies ( $V$ ) finally in terms of due diligence, what are the obligations of the state and what is the limit for this. As conclusion, the authors seek to give a sort of recommendations that will enable both companies to States, effective enforcement of their obligations and responsibilities.
\end{abstract}

Keywords authors: Due diligence, companies, human rights, state responsibility, John Ruggie's guiding principles, global compact, companys role. Keywords plus: Labor contract, human rights, government liability, Ruggie, John Gerard 1944.

\title{
SUMARIO
}

INTRODUCCIÓN.- I. EL PAPEL DE LA EMPRESA EN LA DEBIDA DILIGENCIA EN DEREChos humanos.- $A$. Concepto de debida diligencia en derechos humanos.- B. ¿Cómollevar a cabo la debida diligencia en derechos humanos?.- C. Desarrollo e implementación del proceso de debida diligencia en derechos humanos.- D. Hasta dónde va la responsabilidad de la empresa en materia de derechos humanos: límites de la debida diligencia en los derechos humanos.- II. EL PAPEL DEL ESTAdo EN LA DEBIDA DILIGENCIA EN DERECHOS HUMANOS.- III. FIJANDO EL LÍMITE DE LA ACTUACIÓN ESTATAL.- IV.-CONCLUSIÓN.- BibLIOGRAFÍA. 
INTRODUCCIÓN

Recientemente la Organización de Naciones Unidas creó una relatoría especial para que se encargara de los temas relacionados con las empresas y su relación con los derechos humanos (DDHH); más adelante, el relator John Ruggie elaboró el informe Principios Rectores sobre Empresas y Derechos Humanos, que hoy están dando las pautas de comportamiento a empresas y Estados frente al tema, sin que aún exista un instrumento vinculante a escala internacional que imponga obligaciones a las empresas y no solamente como hasta ahora, deberes y responsabilidades.

Dentro de los principios rectores de Ruggie - que podría afirmarse son un derrotero de buenas prácticas por parte de las empresas frente a los derechos humanos, puesto que sus actividades pueden llegar a afectarlos- , se encuentra el deber de debida diligencia. Este, en pocas palabras, exige que la actuación de las empresas ocurra mediante una consideración previa de todos los riesgos, impactos y posibles vulneraciones que su actividad implica para los DDHH, y que esos riesgos se reduzcan, se eviten o se mitiguen.

No obstante lo anterior y a pesar de que actualmente se vincule la responsabilidad de las empresas, los Estados conservan sus obligaciones frente a la protección, respeto y garantía de los derechos humanos, y por ello dentro de los principios de Ruggie también hay responsabilidades que le competen en exclusividad a los Estados.

A continuación se dan algunas puntadas sobre el concepto de debida diligencia en DDHH, sus implicaciones y límites. Para esto se ofrecerán dos enfoques: el primero desde el papel que desempeñan las empresas en esta materia; el segundo, desde el papel del Estado como garante necesario. 


\section{EL PAPEL DE LA EMPRESA EN LA DEBIDA} DILIGENCIA EN DERECHOS HUMANOS

En el transcurso de los años han ocurrido numerosos ejemplos de empresas relacionadas o participantes en violaciones a los derechos humanos; tales son los casos de la represa de Kariba y en 1979 la represa Sardar Sarovar, por la cual fueron desalojadas más de 6000 familias'; el caso Shell en 1995, cuando activistas como Ken Saro-Wiwa, fueron ejecutados por protestar contra las consecuencias medioambientales y vulneraciones contra la población por esta compañía ${ }^{2}$; el caso de Chiquita Brands condenada por un juez Federal en Estados Unidos, por financiar grupos paramilitares en Colombia ${ }^{3}$, entre otros; de allí que tras un profundo análisis se haya empezado a vincular el tema de la actividad de las empresas con la debida diligencia en materia de DDHH.

\section{A. Concepto de debida diligencia en derechos humanos}

Varias han sido las definiciones dadas al concepto de debida diligencia en materia de derechos humanos. La Iso 26000 por ejemplo señala que se trata de un "proceso exhaustivo y proactivo para identificar los impactos negativos reales y potenciales de carácter social, ambiental y económico de las decisiones y actividades de una organización a lo largo del ciclo de vida completo de un proyecto o de una actividad de la organización, con el objetivo de evitar y mitigar dichos impactos negativos"4 (Resaltado fuera del original).

1 P. Arrojo Agudo, Dir., Agua, ríos y pueblos, 33 (Fundación Nueva Cultura del Agua, Málaga, 2009).

2 The Case Against Shell: the Hanging of Ken Saro-Wiwa. En http://www.youtube.com/ watch?v=htF5XElMyGI (Mayo, 2009). Business \& Human Rights Resource Center. Human Rights Impacts of Oil Pollution: Nigeria. Execution of Ken Saro-Wiwa and Other Activists, and Subsequent Lawsuit Against Shell. En http://www.business-humanrights. org/Documents/Oilpollution/Nigeria/Saro-Wiwa

3 El fallo contra Chiquita Brands: una condena hipócrita. El Turbión (1 de octubre de 2007). En http://elturbion.com/?p=218

4 Organización Internacional de Normalización (Iso,por sus siglas en inglés). Guía de responsabilidad social, 14. ISO/FDIS 26000:2010 (E) ISO/ TMB /WG /SR STTF. En http://www. 
Entonces, según dicha definición, la debida diligencia surgirá ante la conciencia de los posibles daños (o impactos negativos) que cause la mitigación que de ellos se busque. Mark B. Taylor, Luc Zandivliet y Mitra Forouhar miran la debida diligencia en derechos humanos desde una óptica de riesgo. Explican que este concepto procede del us Securities Act de 1993, que proveía a los corredores de Bolsa de Estados Unidos de una defensa cuando eran acusados de haber entregado información confidencial a los inversores; de este modo, podían demostrar que la información faltante no había sido descubierta por ellos, al haber aplicado un método de debida diligencia en la materia. De allí el principio del proceso de investigación debe realizarse con el propósito de prevenir daños ${ }^{5}$ en materia de DDHH.

Según el IPIECA (International Petroleum Industry Environmental Conservation), la debida diligencia en derechos humanos en cuanto a una empresa se refiere al grado de prudencia que esta debe tener en sus operaciones o proyectos, más como un tema de buenas prácticas que como un requisito legal ${ }^{6}$. En este sentido, los principios rectores de las Naciones Unidas sobre empresas y DDHH establecen tres pilares fundamentales:

- "El deber del Estado de proteger los derechos humanos;

- La responsabilidad de las empresas de respetar los derechos humanos;

- La necesidad de mejorar el acceso a las vías de reparación de las víctimas de abusos relacionados con las empresas"7 (Resaltado fuera del original).

Adicionalmente, las Naciones Unidas definen la debida diligencia en derechos humanos como "la medida de prudencia, actividad o asiduidad que cabe razonablemente esperar, y con la que

posgradofadu.com.ar/archivos/biblio_doc/responsabilidad-social.pdf (8 de octubre de 2010).

5 M. Taylor, L. Zandivliet \& M. Forouhar, Due Diligence for Human Rights: a Risk-Based Approach, 2-3 (Harvard University, Working Paper No. 53, Octubre 2009).

6 IPIECA, Proceso de debida diligencia en derechos humanos. Guía práctica de implementación para las empresas de petróleo y gas, 1-11 (Autor, Working Paper, 2012).

7 Organización de Naciones Unidas, La responsabilidad de las empresas de respetar los derechos humanos. Guía para la interpretación. En http://www.ohchr.org/Documents/ Publications/HR.PUB.12.2_sp.pdf (2012). 
normalmente actúa, una [persona] prudente y razonable en unas circunstancias determinadas; no se mide por una norma absoluta, sino dependiendo de los hechos relativos del caso en cuestión" ". En el contexto de los principios rectores se señala:

"La diligencia debida en materia de derechos humanos constituye un proceso continuo de gestión que una empresa prudente y razonable debe llevar a cabo, a la luz de sus circunstancias (como el sector en el que opera, el contexto en que realiza su actividad, su tamaño y otros factores) para hacer frente a su responsabilidad de respetar los derechos humanos"9 (Resaltado fuera del original).

Se adiciona al concepto de debida diligencia el concepto de riesgo en derechos humanos, este último entendido como:

"Se entienden como riesgos de una empresa relacionados con los derechos humanos los riesgos de que sus actividades puedan acarrear una o más consecuencias negativas sobre los derechos humanos. Así pues, guardan relación con su impacto potencial sobre los derechos humanos. En la evaluación del riesgo tradicional, en el riesgo influyen tanto las consecuencias de un suceso (su gravedad) como la probabilidad de que ocurra. En el contexto del riesgo relacionado con los derechos humanos, la gravedad es el factor predominante. La probabilidad puede ser importante a la hora de ayudar a establecer prioridades en el orden en que deben abordarse las posibles consecuencias negativas en determinadas circunstancias (...) Es importante entender que el riesgo de una empresa para los derechos humanos es el riesgo que su actividad plantea para esos derechos. Es distinto del riesgo que la implicación en unas consecuencias negativas sobre los derechos humanos pueda suponer para la empresa, aunque ambos están cada vez más estrechamente relacionados"10 (Resaltado fuera del original).

Según los principios rectores las empresas deben:

- Respetar los derechos humanos en sus proyectos y operaciones.

8 Organización de Naciones Unidas, Óp. cit. Ver también Black's Law Dictionary (6 ${ }^{\text {th }}$ ed., St. Paul, Minnesota, West, 1990).

9 Organización de Naciones Unidas, Ibídem.

10 Ibid, p. 8. 
- Prevenir y mitigar potenciales riesgos a los derechos humanos causados directamente por proyectos y operaciones.

- Tener una política de manejo y prevención de riesgos potenciales a los derechos humanos.

- Expresar públicamente la intención de respetar los derechos humanos.

- Cumplir la debida diligencia en derechos humanos, esto es, hacer la evaluación de riesgos y tomar medidas al respecto.

Según la actividad que desarrolle la empresa, debe remitirse a los convenios de la OIT sobre cada materia. Adicionalmente, otra responsabilidad que estas deben asumir, es no ser cómplice de violaciones o vulneración a los DDHH. Así, se incluye no solo la infracción directa, sino la pasiva.

Por su parte, el CIDSE señala que aunque se le exige a la empresa la debida diligencia en estos aspectos, no debe olvidarse que existen unas "lagunas de gobernanza", es decir, el hecho de que "no todos los Estados están dispuestos o son capaces de cumplir, por sí solos, con su deber de proteger, ya sea por la corrupción de los agentes económicos, la debilidad de las instituciones, o cualquier otra razón"11.

En este sentido, la debida diligencia en derechos humanos es un proceso de gestión continuo, prudente y razonable, que debe hacer la empresa según las variables de la zona donde opera, es decir, el entorno y contexto, con la finalidad de hacer frente a su obligación de respetar los derechos humanos; es un proceso que implica la práctica de prevenir y evitar riesgos potenciales a los DDHH.

Cada una debe hacer un análisis de hechos y datos — que se tratará más adelante - a la luz de los estándares internaciones de protección a los derechos humanos y los parámetros establecidos por las normas nacionales para cada sector.

11 D. Auclair, Diligencia debida en materia de derecho humano: medidas para conseguir una aplicación eficaz. Recomendaciones de CIDSE, 2 (CIDSE, Working Paper, 2013). 
B. ¿Cómo llevar a cabo la debida diligencia en derechos humanos?

Tras tener claro el concepto, cabe preguntar ¡cómo llevarlo a cabo? ¿cuáles elementos y variables tener en cuenta? y ¿cómo desarrollarlo e implementarlo? De acuerdo con los principios rectores de las Naciones Unidas existen cuatro elementos que debe contener el proceso de debida diligencia en derechos humanos:

- Identificar y evaluar impactos potenciales y actuales a los derechos humanos.

- Integrar y actuar sobre los resultados de la evaluación.

- Efectuar seguimiento y monitoreo de la implementación de medidas de prevención y mitigación de los impactos.

- Comunicar y reportar sobre la prevención y mitigación del impacto.

La debida diligencia en DDHH es un proceso en construcción permanente que debe realizarse en todas las etapas de la actividad de la empresa, por tanto, debe implementarse desde el momento en que los proyectos estén en discusión y, por supuesto, también en los que están ya en desarrollo (por ejemplo, cuando se incluye la entrada de nuevo capital, así como en las operaciones y proyectos en curso o en terminación) ${ }^{12}$.

Los riesgos potenciales a los derechos humanos existen en las diversas fases de la operación de la empresa, razón por la cual deben ser identificados en cada etapa y subetapa, y deben contar con seguimiento y monitoreo según una debida diligencia de DDHH. Es importante considerar estos riesgos, sea que le afecten a esta o a la comunidad donde desarrolla su operación, con la finalidad de aplicar buenas prácticas. Este proceso es vital para empresas de gas y petróleo, que operan en regiones donde las condiciones son variables debido a la coyuntura del país o la región, y que pueden mejorar o empeorar con el tiempo.

12 IPIECA, Proceso de debida diligencia en derechos humanos Guía práctica de implementación para las empresas de petróleo y gas, 2 (Autor, Working Paper, 2012). 
Algunos de los estímulos para realizar el proceso de debida diligencia en derechos humanos son: i) La identificación y manejo de los riesgos e impactos potenciales a los derechos humanos en las comunidades donde se desarrolla la operación; ii) Tener en cuenta las condiciones de seguridad del país y de la región respectivos; iii) La prevención de la disrupción de una operación o un proyecto en construcción, para así mejorar el manejo del presupuesto y el cronograma; iv) Mejorar las relaciones con los empleados de la zona y comunidades o grupos de interés, a partir del fomento de la confianza y el diálogo sobre los proyectos y sus implicaciones; v) La protección a los empleados, comunidades y activos de la operación, en cuanto a seguridad del proyecto en temas medioambientales; vi) La creación de contribuciones positivas en la comunidad donde se desarrolla el proyecto, sobre la base de la estrategia de valor compartido; vii) Proteger la reputación de la empresa tanto a escala nacional como internacional; viii) Tratar los abusos potenciales a los derechos humanos o su percepción; ix) Tratar los riesgos potenciales derivados de la asociación derivada de los contratos que tenga la empresa con sus proveedores y contratistas; $x$ ) Realizar una articulación entre las situaciones de la empresa y los estándares en derechos humanos establecidos por los tratados internacionales, tales como los Principios Voluntarios en Seguridad, el Pacto Global, los lineamientos de empresas multinacionales, entre otros; y vi) La necesidad de integrar los sistemas de gestión de la empresa con los sistemas de gestión y manejo de derechos humanos.

\section{Desarrollo e implementación del proceso de debida diligencia en derechos humanos}

Diversas metodologías se han implementado para llevar a cabo el proceso de debida diligencia, bien desde esta esfera de influencia hasta un complejo proceso de verificación de cada proceso y procedimiento, de cada paso en el desarrollo de la operación y actividad de la empresa. Algunas iniciativas muy interesantes se describen a continuación. 
El Global Business Initiative on Human Rights (GBIHR) elaboró un Marco de Derechos Humanos, que busca la reducción de la violación de los DDHH por parte de las empresas mediante seis pasos: i) Caso de empresa mundial: se pretende conocer el concepto de derechos humanos y la vinculación con la empresa; ii) Estrategia: averiguar qué está haciendo la compañía en materia de derechos humanos; iii) Políticas: determinar dónde está contenido el tema de los derechos humanos en las políticas vigentes; iv) Procesos y procedimientos: establecer la base de los derechos humanos en la empresa; v) Capacitación: vincular la materia de derechos humanos a la cultura de la empresa; y vi) Medición y desempeño: establecer indicadores ${ }^{13}$.

El IPIECA por su parte ha diseñado una metodología de manejo del proceso de debida diligencia en DDHH, que incluye seis componentes nucleares:

- Visión y objetivo: la visión y objetivos de la empresa deben estar articulados con el manejo que se pretende dar a los derechos humanos, es decir, deben encajar y estar alineados la política de derechos humanos, el código de conducta, la política y estrategia de responsabilidad social corporativa y otros mecanismos formales.

- Rendición de cuentas: "Dado que las cuestiones de derechos humanos son comunes a distintos departamentos de la empresa, se asignan las funciones y responsabilidades apropiadas, entre ellas, la rendición de cuentas" 14 .

- Evaluación y planificación: incluye "i) identificar las fases en el ciclo de vida del proyecto; (ii) realizar un inventario de los procesos, programas y herramientas existentes (...) que se pueden utilizar para evaluar problemas e impactos potenciales; (iii) recopilar y analizar información de evaluaciones previas o de fuentes externas; $y$ (iv) llevar a cabo

13 Marco de Derechos Humanos. Global Business Initiative on Human Rights (GBIHR). En http://www.global-business-initiative.org/wp-content/uploads/2012/07/Human-Rights-Framework-Poster-Spanish.pdf (2012).

14 IPIECA, Op. cit., p. 5. 
la evaluación para identificar, definir el alcance y analizar problemas e impactos potenciales"15.

- Implementación: una vez identificados y priorizadas las cuestiones e impactos potenciales, los resultados deben ser incorporados en el plan de manejo, lo que incluye la comunicación con los grupos de interés internos y externos, cuando ello sea necesario, lo que permite solucionar cada cuestión e impacto.

- Revisión: se deben establecer una serie de indicadores para hacer seguimiento y evaluar el plan en la fase de implementación. Los indicadores informan sobre la efectividad del proceso y proveen oportunidades de mejoramiento.

- Mejoramiento: concluida la fase de revisión, es importante para proceder a hacer un mejoramiento en el plan y procedimientos, inclusive los procesos internos.

Según Mark B. Taylor, Luc Zandivliet y Mitra ${ }^{16}$, para realizar una debida diligencia en derechos humanos se deben atender dos fases: i) identificación de hechos y datos relevantes y ii) evaluación de esos datos y hechos bajo los estándares de DDHH. Se dice que es necesario incluir en la matriz de riesgos de la empresa la variable de derechos humanos, para así poder hacer el análisis en conjunto. Estas dos fases de materializan mediante nueve pasos: i) Conocer a profundidad el lugar donde se desarrolla la operación; ii) Realizar un mapa o una lista de las operaciones que tiene la empresa; iii) Realizar un chequeo cruzado entre los pasos uno y dos para ver cuáles conexiones existen; iv) Elaborar una lista de riesgos potenciales; v) Efectuar reuniones con el personal de la empresa para evaluar la lista de riesgos potenciales; vi) Establecer contacto con expertos externos conocedores de la situación de riesgos en derechos humanos; vii) Realizar reuniones y visitas a terreno; viii) Ejecutar visitas y reuniones con los grupos de interés; y ix) Convocar reuniones con stakeholders

16 M. Taylor, L. Zandivliet \& M. Forouhar, Óp. cit. 
externos tales como Gobierno, fuerzas militares, ONG, etc., del entorno.

El análisis de toda esta información arrojará cuáles han sido los riesgos encontrados y cuáles las oportunidades de mejoramiento, que ayudarán a la elaboración de un plan de manejo de los riesgos de forma priorizada. De la prudencia, especificidad y conciencia con que se realice el proceso de debida diligencia podrá cumplirse el objetivo, la prevención y mitigación de potenciales violaciones a los DDHH por parte de la empresa.

D. Hasta dónde va la responsabilidad de la Empresa en materia de derechos humanos: límites de la debida diligencia en los derechos humanos

El papel de las empresas y hasta dónde vaya el límite de la debida diligencia en derechos humanos es un tema que está directamente vinculado a la responsabilidad y conocimiento en la materia. Para algunos autores existen diferentes niveles de responsabilidad, entre estos ${ }^{17}$ :

Un primer nivel llamado "omisión con conocimiento", o "dejar pasar dejar hacer", que se materializa cuando una empresa permite que otra viole los DDHH, lo que implicaría que esta empresa también es responsable por la transgresión, aunque su responsabilidad depende del nivel de autonomía otorgada. Según esta teoría, entre mayores ingresos obtenga la empresa por la ejecución de la actividad violatoria de derechos humanos, mayor resulta su responsabilidad.

Un segundo nivel ha sido llamado el "efecto causal" o "bola de nieve", es decir, si la acción de la empresa constituye un efecto causal para que otra cometa violación de derechos humanos o implique la violación del derecho humano. Sería entonces la empresa totalmente responsable cuando la violación cometida por el proveedor está relacionada de forma directa con la empresa solicitante; ejemplo de ello es lo que sucede en la cadena de valor

17 M. Jungk, The Human Rights \& Business Project, Danish Institute for Human Rights, 3-11 (The Human Rights \& Business Project, Working Paper, 2006). 
de la empresa: en la relación con proveedores y subcontratistas es muy importante no resultar involucrados en la responsabilidad por la violación de DDHH de los proveedores. En algunos casos, estos dos principios o niveles implican complicidad.

Un tercer nivel llamado "violación grave" o "severidad de la acción" refiere a una violación directa de la empresa de forma grosera, por ejemplo, aquellas que financian grupos terroristas acusados de genocidios, o las que tienen políticas como las aplicadas en el caso Shell de los años noventa.

Según estos niveles posibles de posible responsabilidad empresarial, es necesario no perder de vista la importancia del acompañamiento y el papel del Estado, lo cual se desarrollará más adelante. Al tener claro el concepto de la debida diligencia en derechos humanos y entender cómo debe llevarse a cabo, es preciso conocer no solo hasta dónde alcanza la responsabilidad de la empresa sino, además, dónde empieza a ser relevante el papel del Estado, como garante del respeto y la protección de los DDHH.

Los principios rectores de derechos humanos y empresas de las Naciones Unidas enfatizan la distinción entre el papel del Estado y el de la empresa. En el análisis presentado anteriormente sobre el desarrollo del proceso de debida diligencia, todos los autores coinciden en determinar la relevancia del contexto, pues resulta vital atender al lugar geográfico donde se ubican la empresa y su operación o donde planea ejecutarla: cada territorio tiene sus propias características y complejidades, razón por la cual es importante que la empresa tenga en cuenta la situación y verifique el papel que el Estado cumple en ese territorio.

Son variados los casos en que se presenta dualidad entre el papel del Estado y el papel de la empresa en cuanto al respeto y promoción de los derechos humanos. Por ejemplo, lo sufrido por la comunidad afrodescendiente de Alto Mira y Frontera, en el municipio de Tumaco, departamento de Nariño, inmersa en un conflicto de tierras, donde convergen empresas sembradoras de palma de aceite, compañías mineras, proyectos de infraestructura y cultivos ilícitos. La comunidad arraigada en la zona desde 
hace varias generaciones, denuncia que el despojo de tierras se inició con las primeras explotaciones de palma de aceite.

La Ley 70 de 1993 estableció la posibilidad de "obtención de derechos de propiedad colectiva sobre tierras baldías del Estado para las comunidades afrocolombianas que históricamente habitaran ese territorio". Pero, una vez que el Consejo Comunitario de Alto Mira y Frontera presentó su solicitud, se encontró con que las compañías de palma de aceite ya habían solicitado esas mismas tierras; de esta manera, de "un lado de la ley quedaron las comunidades que habitan la cuenca desde hace varias generaciones, y del otro la inversión de empresas en el cultivo de tardio rendimiento" $"$.

Contrastan así las versiones dadas por la empresa sobre creación de empleos en la zona, y la denuncia de las comunidades que habitan allí sobre la pérdida de espacio físico, impacto medioambiental al cambiar el uso de la tierra, implicaciones de los monocultivos ${ }^{19}$, y la amenaza a la seguridad alimentaria de la población, al remplazar la palma de aceite a las especies nativas. El 19 de diciembre de 2008, el representante del Ministerio de Agricultura hizo entrega del documento que otorgaba un título colectivo a la comunidad, pero esto fue solo un acto simbólico ya que nunca se hizo entrega formal de las tierras debido a la demanda incoada por la compañía palmicultora ${ }^{20}$.

Situación similar vivieron las comunidades afrocolombianas en las cuencas de los ríos Curvaradó y Jiguamiandó, ubicadas en el bajo Atrato, departamento del Chocó, las cuales se vieron inmersas en violencia, despojo y desplazamiento debido a la llegada de empresas para explotar la palma de aceite y también la instalación del paramilitarismo. Estas circunstancias se aña-

18 T. Alfonso Sierra, L. Grueso Castelblanco, M. Prada Rivas \& Y. Salinas Abdala, Derechos enterrados. Comunidades étnicas y campesinas en Colombia, nueve casos de estudio, 30 (Universidad de los Andes, Centro de Investigaciones Sociojurídicas, Bogotá D. C., 2011).

19 Programa de las Naciones Unidas para el Desarrollo. Historias para contar. Tumaco reclama atención. En http://www.pnud.org.co/sitio.shtml?apc=1l--1--\&x=53528 (2008).

20 T. Alfonso Sierra et ál., Óp. cit., p. 43. 
dieron a la falta de presencia del Estado y la no aplicabilidad de la Ley 70 de 1993 debido al conflicto armado ${ }^{21}$.

El análisis que se realice en el desarrollo del proceso de debida diligencia por parte de las empresas necesariamente debe incluir la Ley y sus implicaciones en cuanto al desarrollo de sus actividades. Algunas normas y leyes expedidas por el Estado pueden generar un conflicto entre las comunidades y las empresas. Para estas consecuencias negativas se debe buscar un punto de equilibrio tripartito, en el cual la empresa, las comunidades y el Estado trabajen de forma conjunta en la protección y promoción de los derechos humanos de la comunidad. El Estado debe estar allí para dar un lineamiento claro a las empresas sobre cómo respetar los derechos de las comunidades.

Vale la pena mencionar un caso dramático: en 2007 el Gobierno impulsó la estrategia de desarrollo denominada Alianza Guapi - que pretendía alianzas entre empresarios de palma de aceite con cinco consejos comunitarios reconocidos - regulada por el documento Conpes 3491, pero únicamente uno de los consejos comunitarios aceptó unirse. El resto no quiso participar debido a los efectos de seguridad alimentaria que, consideraron, traería el monocultivo de palma de aceite. "Hasta el momento, ningún operador del sector empresarial se ha vinculado al proyecto para conformar la concebida asociación. Por su alta humedad y pluviosidad, la zona es particularmente vulnerable a las enfermedades de la palma"22.

La falta organización, estudio, coordinación y valoración de las implicaciones y riesgos por parte del Estado, al diseñar una estrategia sin consultar a la población sobre sus necesidades, generaron un contexto vulnerable y facilitaron la violación de DDHH. A continuación algunas puntadas de la obligación del Estado en esta materia así como de las empresas bajo la perspectiva de la debida diligencia.

21 Ibídem, p. 47.

22 Ibíd., p. 67. 


\section{El PAPEL DEL ESTADO EN LA DEBIDA DILIGENCIA EN DERECHOS HUMANOS}

Tradicionalmente ha recaído en los Estados la responsabilidad de combatir y evitar las agresiones a los DDHH, en tanto que son los Estados los que, como sujetos de derecho internacional, ratifican los tratados internacionales de DDHH y se obligan a cumplir lo allí dispuesto, bajo cuyo mandatos su actuar deviene calificado.

Sin embargo, en las dinámicas económicas del mundo de hoy, la responsabilidad de actuar conforme a los preceptos que en materia de DDHH existen, ya no es exclusiva del Estado, sino que es necesario que se vea a los terceros particulares como actores fundamentales en esta materia.

Así las cosas, se debe resaltar que los principios rectores de las Naciones Unidas sobre empresa y derechos humanos incluyen varias esferas del tratamiento de los DDHH, en este sentido, se especifica que es deber del Estado proteger, respetar y garantizar los derechos humanos, y el deber de la empresa es respetarlos y no ser cómplice de violaciones estos.

"Estos Principios Rectores se basan en el reconocimiento de: a) Las actuales obligaciones de los Estados de respetar, proteger y cumplir los derechos humanos y las libertades fundamentales; $b$ ) El papel de las empresas como órganos especializados de la sociedad que desempeñan funciones especializadas y que deben cumplir todas las leyes aplicables y respetar los derechos humanos; c) La necesidad de que los derechos y obligaciones vayan acompañados de recursos adecuados y efectivos en caso de incumplimiento" 23 (Resaltado fuera del original).

Tanto tratados internacionales, jurisprudencia internacional y cortes nacionales han tratado las obligaciones de los Estados frente a la protección y respeto de DDHH. La Convención Americana de Derechos Humanos (en adelante CADH) y la Decla-

23 Organización de Naciones Unidas, Informe del representante especial del Secretario General para la cuestión de los derechos humanos y las empresas transnacionales y otras empresas. Principios Rectores sobre las empresas y los derechos humanos: puesta en práctica del marco de las Naciones Unidas para "proteger, respetar y remediar". Anexo. A/HRC/17/31. Distr. General (21 de marzo de 2011). 
ración Universal de Derechos Humanos ${ }^{24}$, hacen referencia en sus preámbulos a la obligación que tienen los Estados de actuar como garantes de los DDHH. El Artículo 1 de la CADH notifica la obligación de los Estados de prevenir, investigar y sancionar las violaciones de $\mathrm{DDHH}^{25}$.

Por su parte, la jurisprudencia de la Corte Interamericana de Derechos Humanos (en adelante CIDH) ha establecido que el Estado, como garante, tiene la obligación de proteger los DDHH y prevenir violaciones que puedan darse como consecuencia de las actividades de terceros particulares ${ }^{26}$.

"La Corte también ha reconocido que puede generarse responsabilidad internacional del Estado por atribución a éste de actos violatorios de derechos humanos cometidos por terceros o particulares, en el marco de las obligaciones del Estado de garantizar el respeto de esos derechos entre individuos. En este sentido, este Tribunal ha considerado que dicha responsabilidad internacional puede generarse también por actos de particulares en principio no atribuibles al Estado [Las obligaciones erga omnes de respetar y hacer respetar las normas de protección, a cargo de los Estados Partes en la Convención] proyectan sus efectos más allá de la relación entre sus agentes y las personas sometidas a su jurisdicción, pues se manifiestan también en la obligación positiva del Estado de adoptar las medidas necesarias para asegurar la efectiva protección de los derechos humanos en las relaciones inter-individuales. La atribución de responsabilidad al Estado por actos de particulares puede darse en casos en que el Estado incumple, por acción u omisión de sus agentes cuando se encuentren en posición de garantes, esas obligaciones erga omnes contenidas en los artículos 1.1 y 2 de la Convención" 27.

24 Declaración Universal de Derechos Humanos, 1948. Preámbulo. "Los Estados Miembros se han comprometido a asegurar, en cooperación con la Organización de las Naciones Unidas, el respeto universal y efectivo a los derechos y libertades fundamentales del hombre".

25 Convención Americana de Derechos Humanos. "Artículo 1. Los Estados Partes en esta Convención se comprometen a respetar los derechos y libertades reconocidos en ella y a garantizar su libre y pleno ejercicio a toda persona que esté sujeta a su jurisdicción, sin discriminación alguna por motivos de raza, color, sexo, idioma, religión, opiniones políticas o de cualquier otra índole, origen nacional o social, posición económica, nacimiento o cualquier otra condición social".

26 CIDH. González y otras ("Campo algodonero") vs. México. Excepciones preliminares, fondo, reparaciones y costas. Sentencia Serie C No. 205. páras. 234, 243 y 252 (16 de noviembre de 2009).

27 CIDH. Masacre del Pueblo de Bello vs. Colombia. Fondo, reparaciones y costas. Sentencia Serie C No. 140., pár. 113 (31 de enero de 2006). 
En el caso colombiano, la Corte Constitucional en las sentencias T-348 de 2012, T-824 de 2007 y T-769 de 2009 28 , ha establecido de cierta forma que el Estado, en cabeza de las autoridades competentes según la materia, debe encargarse de verificar que se cumplan con todos los requisitos necesarios para evitar que ocurran afectaciones a los derechos humanos de las personas y comunidades que pueden ser impactados por las actividades empresariales.

Si nos centramos en el tema de debate de este artículo, si bien las empresas tienen el deber de actuar con debida diligencia, ello no puede llevar jamás a la idea equivocada de que cuando hay un tercero en escena, el Estado se puede desligar de sus obligaciones. Es claro que el Estado debe exigir y vigilar que las empresas en efecto actúen con debida diligencia, en especial, cuando las actividades son riesgosas en potencia para los DDHH. A este respecto, los comentarios para la interpretación del principio $4^{\mathrm{O}}$ de los Principios Rectores sobre las Empresa y los Derechos Humanos de Ruggie, señalan:

"Los Estados, a título individual, son los principales sujetos de las obligaciones conforme a las normas internacionales de derechos humanos y, colectivamente, los garantes del régimen internacional de derechos humanos. Cuando una empresa está controlada por el Estado o cuando sus actos pueden atribuirse por alguna otra razón al Estado, una violación de los derechos humanos por esta empresa puede implicar una violación de las obligaciones conforme al derecho internacional del propio Estado"29.

El Estado tiene la obligación de hacer seguimiento a las empresas para que estas actúen bajo la debida diligencia, y ello es posible cuando existen normas, requisitos para el funcionamiento, sistemas de monitoreo y seguimiento, entre otras medidas que lo evidencien; además exige un sistema judicial eficiente que sancione a quienes incumplen con tales deberes.

\footnotetext{
28 Corte Constitucional de Colombia. Sentencia T-348 de 2012. (Magistrado Ponente Jorge Ignacio Pretelt Chaljub, 15 de mayo de 2012); Sentencia T-824 de 2007. (Magistrado Ponente Jaime Córdoba Triviño, 05 de octubre de 2007); Sentencia T- 769 de 2009. (Magistrado Ponente Nilson Pinilla Pinilla, 20 de octubre de 2009).

29 Organización de las Naciones Unidas, Óp. cit.
} 


\section{A. Fijar el límite de la actuación estatal}

Con lo dicho hasta este punto, vale preguntarse ¿hasta dónde va el límite de lo exigible para los Estados en materia de debida diligencia? Quizá lo primero que deba decirse es que esta es una responsabilidad de las empresas y no del Estado, pero lo que sí se le exige al Estado es que mediante las medidas que elija demuestre que les ha impuesto a las compañías el deber de cumplir los preceptos de la debida diligencia y que, de no hacerlo, habrá sanciones o implicaciones.

"[E]l deber de los Estados de proteger contra las violaciones de los derechos humanos implica que estos "deben adoptar las medidas apropiadas para prevenir, investigar, castigar y reparar esos abusos mediante políticas adecuadas, actividades de reglamentación y sometimiento a la justicia" ${ }^{30}$. Esto incluye una serie de medidas, desde ofrecer orientación efectiva a las empresas sobre cómo respetar los derechos humanos, hasta garantizar el cumplimiento de las leyes establecidas que exigen a las empresas respetar los derechos humanos. También se incluyen medidas que el Estado puede utilizar para influir en el comportamiento de diversos actores económicos, ya sean reguladores, compradores, inversores o propietarios" $" 31$.

Es importante que el Estado y las instituciones que lo representan, manejen un discurso uniforme y coherente, sin olvidar que el Estado debe ser el primero en evitar la ocurrencia de violaciones a DDHH en su territorio.

En el caso colombiano existe una consagración constitucional del desarrollo sostenible ${ }^{32}$ y de la participación de las comunidades en la decisión de proyectos que puedan afectarlos ${ }^{33}$. Estas normas suelen, en la práctica, entrar en conflicto con políticas de desarrollo como la actual política minera, alrededor de la cual es inevitable que haya aversión de las comunidades a la exploración y explotación minera ${ }^{34}$.

30 Organización de las Naciones Unidas, Ibidem, principio 1.

31 O. De Schutter, A. Ramasastry, M. Taylor \& R. Thompson, La diligencia debida en materia de derechos humanos: el papel de los Estados, 8 (ICAR, ECCJ, CNCARCRCE, CIDSE, 2012).

32 Constitución Política de Colombia, Artículo 80.

33 Óp. cit., Artículo 330.

34 Alfonso Sierra, et ál., Óp. cit., pp. 20 y ss. 
En casos como el referido, el Estado deberá, tal y como lo ha dicho la Corte Constitucional ${ }^{35}$, cerciorarse de que las empresas cumplan con el requisito de consulta previa ${ }^{36}$, e incluso ha dicho ese Tribunal que se trata de una obligación del Estado y no puede verse como un mero requisito que se satisface solo informando a la comunidad: debe ser un espacio de real participación y el garante de ello no es otro que el Estado.

En ese mismo sentido, la Corte Interamericana, a propósito del caso Saramaka vs. Surinam, estableció como obligación estatal la realización de la consulta previa y la obtención de consentimiento informado, en aquellos casos en que se piensa realizar proyectos de desarrollo e inversión que, de una u otra forma, puedan llegar a afectar a las comunidades ${ }^{37}$.

El papel del Estado en este tema no se agota al otorgar las licencias y permisos para que la empresa entre en funcionamiento, simplemente el papel cambia y cuando la empresa inicia actividades, el rol estatal, podemos decir, es el de un guardián. El Estado deberá dejar claro que las actuaciones empresariales son monitoreadas y que ante cualquier desviación habrá una sanción o implicación negativa.

Si bien es claro que existe el deber para toda empresa de actuar diligentemente, los Estados tampoco pueden excusarse en ello para no actuar, estos deben dar la pauta de comportamiento, en especial, cuando se trata de empresas que no son nacionales que deben tener claro el estándar existente de protección de derechos humanos. Infortunadamente, la práctica ha demostrado que empresas nacionales de países con un alto estándar de protección de derechos humanos, llegan a países con legislaciones débiles y terminan por incurrir en conductas que ponen en riesgo y vulneran los derechos de quienes trabajan para ellos o de las comunidades aledañas.

35 Corte Constitucional de Colombia. Sentencia T-348 de 2012 (M. P. Jorge Ignacio Pretelt Chaljub, 15 de mayo de 2012).

36 Corte Constitucional de Colombia. Sentencia T-129 de 2011 (M. P. Jorge Iván Palacio Palacio, 03 de marzo de 2011).

37 CIDH. Pueblo Saramaka vs. Surinam. Excepciones preliminares, fondo, reparaciones y costas. Sentencia Serie C No. 172, páras. 133 a 137 (28 de noviembre de 2007). 
Un ejemplo reciente ocurrió a inicios de 2013 en Bangladesh ${ }^{38}$, donde colapsó la estructura en que funcionaba una fábrica textil, la cual proveía a varias cadenas de ropa europeas. En los países donde esas empresas son nacionales, una situación así hubiese sido poco probable, y de haber sucedido ya existiría una indemnización para las víctimas, contrario a lo que ocurre en Bangladesh. Más allá de la responsabilidad de las empresas involucradas aunque sin relevarlas, es absolutamente preocupante la ausencia estatal en este caso, sumado a lo difícil que resulta para los tribunales nacionales juzgar a los inversionistas extranjeros.

Como se señala en el informe liderado por el profesor Oliver de Schutter, es importante que los Estados garanticen que sus sistemas jurídicos: i) Van a permitir declarar la responsabilidad penal de las empresas; ii) Deben garantizar que su sistema jurídico permite declarar la responsabilidad civil; y iii) Deben garantizar que por medio de reglamentación administrativa se genere la obligación de las empresas de responder ante autoridades administrativas por las faltas que ocurran en contra de los DDHH ${ }^{39}$.

En el caso colombiano existen ciertas circunstancias que deben ser tenidas en cuenta y que van a ser determinantes en el papel que las empresas deben asumir frente a los derechos humanos. Colombia es uno de los países con mayor biodiversidad del mundo y cuenta con aproximadamente 102 pueblos indígenas ${ }^{40}$. Para 2005, el DANE censó a 1392623 indígenas y a 4311757 afrodescendientes en el territorio nacional ${ }^{41}$. Si, adicionalmente, consideramos que se trata de un país en conflicto, con un sinnúmero de grupos ilegales y bandas criminales, con una elevada

38243 muertos en el siniestro en una fábrica textil de Bangladesh. El País. En http://internacional.elpais.com/internacional/2013/04/25/actualidad/1366907042_936828.html (25 de abril de 2013)

39 De Schutter et ál., p. 73.

40 Organización Nacional Indígena de Colombia [ONIC], Pueblos indígenas ¿cuáles son, cuantos y donde se ubican los pueblos indigenas de Colombia? En http://cms.onic.org.co/ pueblos-indigenas/

41 Departamento Administrativo Nacional de Estadística [DANE]. Colombia una nación multicultural. Su diversidad étnica, 37 (Autor, Bogotá, 2007). En http://www.dane.gov. co/files/censo2005/etnia/sys/colombia_nacion.pdf 
actividad de redes narcotraficantes en todo el territorio, y con un conflicto de generaciones por temas de tierras y violaciones a los derechos humanos, se está frente a un contexto complejo que debe ser considerado por las empresas.

Así, por ejemplo, la primera consideración que debe hacerse es la relacionada con las comunidades indígenas, además de otra serie de comunidades que existen y respecto de las cuales, la Corte Constitucional se ha referido en múltiples oportunidades, sobre todo, en lo concerniente a su derecho fundamental a la consulta previa ${ }^{42}$. Las empresas cuyas actividades puedan tener algún tipo de repercusión en las dinámicas de vida de estos grupos de población, deben ser muy cuidadosas y generar el menor impacto posible, pero con el apoyo y acompañamiento de un Estado fuerte que otorgue garantías a ambas partes. Las empresas deben saber y conocer que existe una concepción constitucional del desarrollo sostenible, de la protección del medioambiente, entre otras que también les competen ${ }^{43}$ y que, por ende, exigen que sus actividades y comportamientos se ajusten a estas.

Si bien la Corte Constitucional ha sido bastante proactiva en la defensa y protección de los derechos humanos al exigir que tanto empresas como Estado respondan por las afectaciones, existen políticas desde el Ejecutivo que no están coordinadas entre los diferentes entes locales, municipales y nacionales; esto ha causado que se generen situaciones en que el Estado desconoce por completo lo que la empresa hace y sus consecuencias. Es recurrente conocer casos donde la entrada en funcionamiento de una compañía cumple los requisitos de forma satisfactoria, fase en la cual el Estado está absolutamente presente, pero luego esta misma instancia olvida el acompañamiento y deja la puerta abierta a la comisión de irregularidades por parte de las empresas.

Es lamentable que una vez cumplidos los requisitos, muchas veces solo formales, la el ente estatal abandone tanto a la empresa

\footnotetext{
42 Corte Constitucional de Colombia. Sentencia T-348 de 2012 (M. P. Jorge Ignacio Pretelt Chaljub, 15 de mayo de 2012).
}

43 Alfonso Sierra et ál., Ibídem. 
como a las comunidades: a las empresas no les muestra dónde están los límites de sus actuaciones, y las comunidades quedan sin que la entidad vele por sus derechos humanos.

Entonces, para dar respuesta a la pregunta que nos planteamos, puede afirmarse que el Estado no tiene un límite, sino que cuando hay un tercero, en este caso las empresas, debe velar porque estas respeten los derechos humanos, por cuanto bajo ninguna circunstancia la instancia estatal está eximida de cumplir con sus obligaciones. 


\section{CONCLUSIÓN}

Bajo los parámetros de los Principios Rectores sobre Empresas y Derechos Humanos es claro que el Estado tiene tres niveles de obligaciones frente al tema de los derechos humanos, proteger, respetar y reparar. Por otra parte, la empresa tiene una responsabilidad frente a los derechos humanos: respetar.

Para asumir esta responsabilidad es necesario que la empresa cumpla un proceso de debida diligencia y basada en el contexto, identifique elementos, componentes e indicadores específicos para conocer los riegos e impactos de su actividad y operación, que en gran cantidad de casos ni siquiera imaginan. De allí que la empresa pueda hacer un plan y priorizar cuáles de estos riegos o impactos tratará primero. El proceso de debida diligencia, genera además buenas prácticas dentro de la organización y un entorno de armonía para las comunidades y usuarios.

Sin embargo, la empresa tiene su actividad y opera en un territorio específico, por ende tiene un Estado soberano que debe ser capaz de ofrecer las herramientas y el acompañamiento necesario para que el proceso de debida diligencia empresarial no quede en una simple iniciativa.

Se recomienda al Estado, como garante de los derechos humanos de sus ciudadanos, que trabaje de la mano con las empresas y que cuando firme y ratifique los Tratados de Libre Comercio tenga en cuenta la variable de derechos humanos de sus habitantes; solo así podrá advertir las condiciones bajo las cuales puede operar una nueva empresa en el territorio colombiano, en un marco de beneficios para esta y para todos los habitantes. 
BIBLIOGRAFÍA

Libros

Alfonso Sierra, T., Grueso Castelblanco, L., Prada Rivas, M. \& Salinas Abdala, Y., Derechos enterrados comunidades étnicas y campesinas en Colombia, nueve casos de estudio, 30 (Universidad de los Andes, Centro de Investigaciones Sociojurídicas, Bogotá D. C, 2011).

Arrojo Agudo, P. (Dir.), Agua, ríos y pueblos, 33 (Fundación Nueva Cultura del Agua, Málaga, 2009).

De Schutter, O., Ramasastry, A., Taylor, M. \& Thompson, R., La diligencia debida en materia de derechos humanos: el papel de los Estados, 8 (ICAR, ECCJ, CNCARCRCE, CIDSE, 2012).

\section{Jurisprudencia}

Corte Interamericana de Derechos Humanos [CIDH]. González y otras (“Campo algodonero") vs. México. Excepciones preliminares, fondo, reparaciones y costas. Sentencia Serie C No. 205, páras. 234, 243 y 252 (16 de noviembre de 2009).

CIDH. Pueblo Saramaka vs. Surinam. Excepciones preliminares, fondo, reparaciones y costas. Sentencia Serie C No. 172, páras. 133 a 137 (28 de noviembre de 2007).

CIDH. Masacre del Pueblo de Bello vs. Colombia. Fondo, reparaciones y costas. Sentencia Serie C No. 140, pár. 113 (31 de enero de 2006).

Corte Constitucional de Colombia. Sentencia T-348 de 2012 (M. P. Jorge Ignacio Pretelt Chaljub, 15 de mayo de 2012).

Corte Constitucional de Colombia. Sentencia T- 129 de 2011 (M. P. Jorge Iván Palacio Palacio, 03 de marzo de 2011).

Corte Constitucional de Colombia. Sentencia T- 824 de 2007 (M. P. Jaime Córdoba Triviño, 05 de octubre de 2007).

Corte Constitucional de Colombia. Sentencia T- 769 de 2009 (M. P. Nilson Pinilla Pinilla, 20 de octubre de 2009).

\section{Tratados Internacionales y otros acuerdos internacionales}

Convención Americana de Derechos Humanos, 1969.

Declaración Universal de Derechos Humanos, 1948.

Organización de Naciones Unidas. Informe del representante especial del Secretario General para la cuestión de los derechos humanos y las empresas transnacionales y otras empresas. Principios rectores sobre las empresas y los derechos humanos: puesta en práctica del marco de las Naciones Unidas para "proteger, respetar y remediar”. Anexo. A/HRC/17/31. Distr. General (21 de marzo de 2011). 


\section{Fuentes Digitales}

Business \& Human Rights Resource Center. Human Rights Impacts of Oil Pollution: Nigeria. Execution of Ken Saro-Wiwa and Other Activists, and Subsequent Lawsuit against Shell. En http://www.business-humanrights.org/Documents/ Oilpollution/Nigeria/Saro-Wiwa

Departamento Administrativo Nacional de Estadística [DANE]. Colombia una Nación Multicultural. Su diversidadétnica. 37 (Autor, Bogotá, 2007). En http:// www.dane.gov.co/files/censo2005/etnia/sys/colombia_nacion.pdf

Global Business Initiative on Human Rights (GBIHR) (2012). Marco de derechos humanos. En http://www.global-business-initiative.org/wp-content/uploads/2012/07/Human-Rights-Framework-Poster-Spanish.pdf

Organización Internacional de Normalización (Iso, por sus siglas en inglés). Guía de responsabilidad social, 14. ISO/FDIS 26000:2010 (E) ISO/ TMB /WG /SR STTF. En http://www.posgradofadu.com.ar/archivos/biblio_doc/responsabilidad-social.pdf (8 de octubre de 2010).

Organización Nacional Indígena de Colombia [ONIC], Pueblos indígenas ¿cuáles son, cuantos y donde se ubican los pueblos indigenas de Colombia? En http://cms.onic.org.co/pueblos-indigenas/

Programa de las Naciones Unidas para el Desarrollo [PNUD]. Historias para contar. Tumaco reclama atención. En http://www.pnud.org.co/sitio.shtml?apc=ll-$1--\& x=53528$ (2008).

\section{Prensa}

243 muertos en el siniestro en una fábrica textil de Bangladesh. El País. En http://internacional.elpais.com/internacional/2013/04/25/actualidad/1366907042_936828. html (25 de abril de 2013).

El fallo contra Chiquita Brands: una condena hipócrita. El Turbión En http://elturbion.com/?p=218 (1 de octubre de 2007)

\section{Otros}

Auclair, D., Diligencia debida en materia de derecho humano: medidas para conseguir una aplicación eficaz. Recomendaciones de CIDSE, 2 (CIDSE, Working Paper, 2013).

Black's Law Dictionary (6 ${ }^{\mathrm{TH}}$ ed., St. Paul, Minnesota, West, 1990).

Constitución Política de Colombia, 1991.

IPIECA, Proceso de debida diligencia en derechos humanos. Guía práctica de implementación para las empresas de petróleo y gas, 1-11 (Autor, Working Paper, 2012).

Jungk, M., The Human Rights \& Business Project, Danish Institute for Human Rights, 3-11 (The Human Rights \& Business Project, Working Paper, 2006). 
Taylor, M., Zandivliet, L. \& Forouhar, M., Due Diligence for Human Righst: a RiskBased Approach, 2-3 (Harvard University, Working Paper 53, Octubre 2009).

The Case against Shell: The Hanging of Ken Saro-Wiwa. En http://www.youtube. com/watch?v=htF5XElMyGI (18 de mayo de 2009). 